Heinzelmann, Christoph; Weichert, Roman; Wassermann, Stefanie Hydraulische Untersuchungen zum Bau einer Fischaufstiegsanlage in Lauffen am Neckar

Originalveröffentlichung / Original Publication:

https://www.springerprofessional.de/hydraulische-untersuchungen-zum-bau-einer-fischaufstiegsanlage-i/3416988

Verfügbar unter / Available at:

https://hdl.handle.net/20.500.11970/104559

Vorgeschlagene Zitierweise / Suggested citation:

Heinzelmann, Christoph; Weichert, Roman; Wassermann, Stefanie (2013): Hydraulische Untersuchungen zum Bau einer Fischaufstiegsanlage in Lauffen am Neckar. In: Wasserwirtschaft 103 (1-2). S. 26-32. 
Erstveröffentlichung in WasserWirtschaft 1/2 (2013), S. 26-32.

Für eine korrekte Zitierbarkeit ist die Seitennummerierung

der Originalveröffentlichung für jede Seite kenntlich gemacht.

\title{
Hydraulische Untersuchungen zum Bau einer Fischaufstiegsan- lage in Lauffen am Neckar
}

\author{
Christoph Heinzelmann, Roman Weichert und Stefanie Wassermann
}

Als technisch-wissenschaftlicher Dienstleister für die Wasser- und Schifffahrtsverwaltung des Bundes (WSV) berät die Bundesanstalt für Wasserbau (BAW) die WSV-Dienststellen auch in Fragen der Fischdurchgängigkeitan Bundeswasserstraßen. Dabei konzentrieren sich die Untersuchungen der BAW auf die hydraulischen und wasserbaulichen Aspekte. In diesem Beitrag wird über die laufenden Untersuchungen zum Bau einer Fischaufstiegsanlagean der Neckarstaustufe Lauffen berichtet. Zum Einsatz kommen Naturuntersuchungen sowie physikalische und numerische Modellsimulationen.

\section{Einleitung}

Die Erhaltung und Wiederherstellung der ökologischen Durchgängigkeit an staugeregelten Fließgewässern zählt zu den wichtigsten Umweltzielen der Wasserrahmenrichtlinie (WRRL). Hierbei spielt die Fischdurchgängigkeit, also der Fischaufstieg und Fischabstieg, an den Stauanlagen eine wesentliche Rolle.

Seit Inkrafttreten des novellierten Wasserhaushaltsgesetzes am 1. März 2010 obliegt dem Bund diese Aufgabe an den von der Wasser- und Schifffahrtsverwaltung des Bundes (WSV) errichteten und betriebenenStauanlagen. Eine erste Analyse [1] hat gezeigt, dass die überwiegende Zahl der WSV-Staustufen über keine ausreichend funktionsfähigen Anlagen verfügt. Somit wird an vielen Staustufen der Bau von Fischauf- und Fischabstiegsanlagen bzw. deren Ertüchtigung erforderlich. Dabei sind für Fischauf- und -abstieg in der Regel unterschiedliche Anlagen zu realisieren, da sich die Fische nach dem heutigen Kenntnisstand im Auf- und im Abstiegsfall unterschiedlich verhalten. Gegenwärtig liegt der Fokus der WSV auf den Fragen des Fischaufstiegs. Hier existieren aktuell an ca. 250 Staustufen Defizite, die gemäß den Fristen der WRRL spätestens bis zum Jahr 2027 zu beseitigen sind. Entweder fehlen die Anlagengänzlich oder sie funktionieren nicht zufriedenstellend. Allein für den Neubau und die Ertüchtigung von Fischaufstiegsanlagen (FAA) an Bundeswasserstraßen belaufen sich die Baukosten nach ersten Schätzungen auf ca. 700 Mio. EUR.

In ihrer Funktion als fachwissenschaftliche Dienstleister für die WSV beraten und unterstützen in enger Zusammenarbeit die Bundesanstalt für Wasserbau (BAW) und die Bundesanstalt für Gewässerkunde (BfG) die WSV-Dienststellen in Fragen der ökologischen Durchgängigkeit. Dabei konzentrieren sich die Untersuchungen der BAW auf die hydraulischen und wasserbaulichen Aspekte, während die BfG die biologischen Aspekte untersucht. Eigenes Fachwissen, Fachliteratur und die 


\section{Autorenfassung}

Heinzelmann, Weichert, Wassermann: Hydraul ische Untersuchungen zum Bau einer Fischaufstiegsanlage in Lauffen am Neckar, 2013

Unterstützung durch externe Fachbüros und Forschungseinrichtungen dienen dabei als wichtige Grundlagen. Zur Weiterentwicklung des Stands von Wissenschaft und Technik über FAA werden zudem vertiefte Untersuchungen an Pilotstandorten sowie eigene Forschungs- und Entwicklungsvorhaben durchgeführt. Ziel ist es, neben der Entwicklungvon Ausführungsvorschlägen für konkrete Baumaßnahmen auch grundsätzliche Empfehlungen für Anordnung, Ausrichtung, Dimensionierung, Dotierung, Betriebsweise und Überprüfung von FAA an Bundeswasserstraßen zu erarbeiten.

Die nachfolgenden Ausführungen befassen sich mit den laufenden Untersuchungen der BAW zum Bau einer neuen FAA an der Neckarstaustufe Lauffen. Im

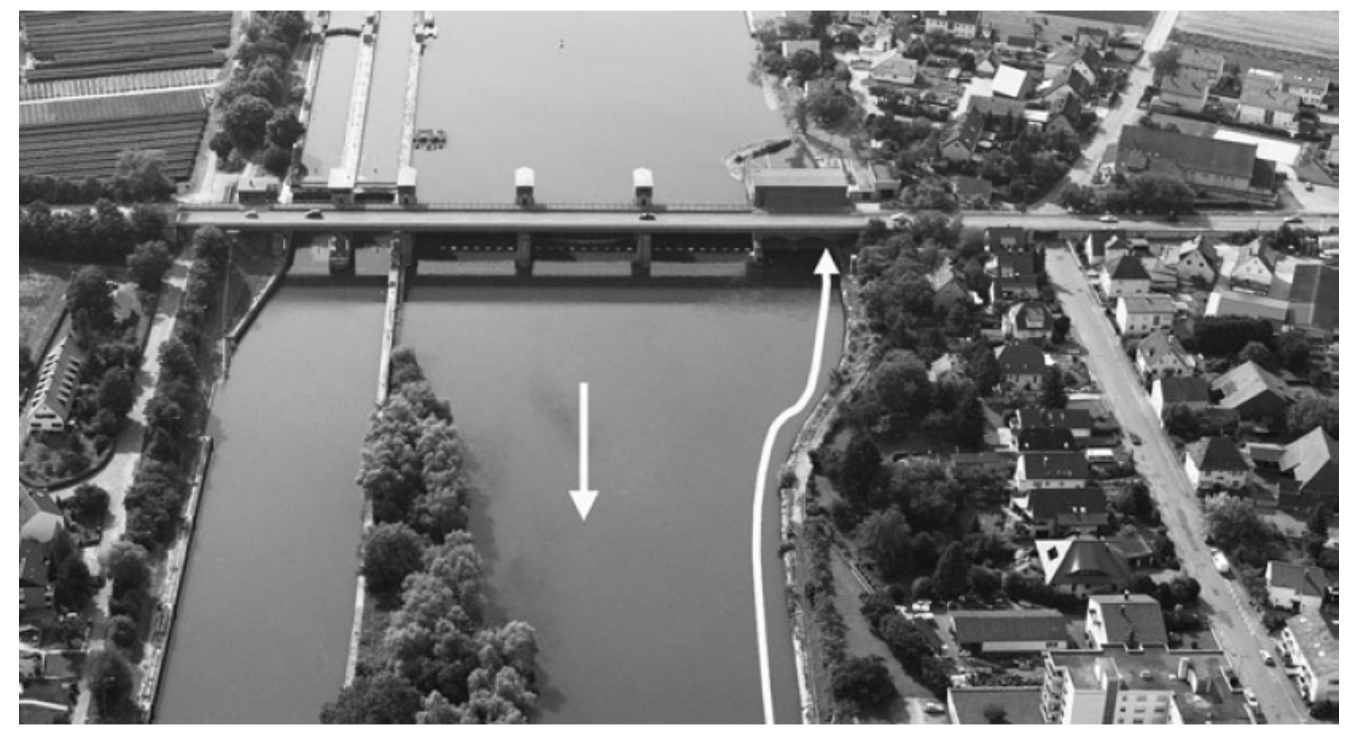

Bild 1: $\quad$ Neckarstaustufe Lauffen, Blick vom Unterwasser (Quelle: Amt für Neckarausbau Heidelberg)

Heinzelmann, Weichert, Wassermann: Hydraulische Untersuchungen zum Bau einer Fischaufstiegsanlage in Lauffen am Neckar. WasserWirtschaft 1/2 (2013), S. 26-32.

Mittelpunkt stehen dabei die hydraulischen Untersuchungen im Unterwasser der Staustufe, die mit Hilfe von Naturmessungen sowie physikalischen und numerischen Modellsimulationen durchgeführt werden. Als Ausblick werden auch die geplanten Modelluntersuchungen für die FAA selbst vorgestellt. 


\section{Autorenfassung}

Heinzelmann, Weichert, Wassermann: Hydraul ische Untersuchungen zum Bau einer Fischaufstiegsanlage in Lauffen am Neckar, 2013

\section{Wissensdefizite für Fischaufstiegsanlagen an Bundeswasserstraßen}

Nationale Regelwerke zum Bau von FAA existieren: z. B. der Gelbdruck des DWAMerkblatts 509 [2] oder das Handbuch Querbauwerke des Landes Nordrhein-Westfalen [3]. Allerdings basieren die dort genannten Richt- und Erfahrungswerte auf Erkenntnissen, die eher an kleinen und mittelgroßen Fließgewässern und nicht an großen Gewässern, wie sie typisch für Bundeswasserstraßen sind, gewonnen wurden. Demzufolge bestehen insbesondere an Bundeswasserstraßen noch größere Wissensdefizite für FAA. Auch die etwa 60 internationalen Regelwerke, die im Rahmen einer Literaturstudie [4] ausgewertet wurden, können diese Lücken nicht schließen.

Die Funktionsfähigkeiteiner FAA hängt maßgeblich von zwei Faktoren ab: der Auffindbarkeit der Anlage im Unterwasser einer Staustufe und der Passierbarkeit der Anlage. Die Wissensdefizite bezüglich der Auffindbarkeit umfassen z. B. die Gestaltung des Einstiegs oder die Frage, wie der Turbulenzgrad das Fischverhalten beeinflusst. Die Fragen, wie die Fische auf konkurrierende Strömungen reagieren, wie die Auffindbarkeit bei komplexen Anlagen mit mehreren Gewässerarmen zu realisieren ist, wie sich wechselnde Wasserstände auswirken oder welchen Beitrag Schiffsschleusen leisten können, zeigen die typischen Besonderheiten, die bezüglich der Auffindbarkeit an Bundeswasserstraßen zu beachten sind. Die Wissensdefizite bezüglich der Passierbarkeit umfassen vor allem Aspekte der geometrischen und hydraulischen Dimensionierung der Anlage und deren Wirkung auf das Fischverhalten.

Die Erkenntnisse, die an bestehenden FAA gewonnen wurden, sowie theoretische Überlegungen zeigen, dass bei den Fischen, die regelmäßig über eine oder mehrere Staustufen wandern müssen, eine Bestandserhaltung und -entwicklung im Regelfall nur gelingt, wenn ein sehr großer Anteil der Fische die FAA ohne Zeitverzögerung finden und überwinden kann. Folglich genügt es nicht, wenn FAA grundsätzlich für die betreffenden Fischarten passierbar sind. Sie müssen ebenso zügig überwunden werden können, um ökologisch wirksam zu sein. Darüber hinaus wird gefordert, dass die Funktionsfähigkeit der Anlage an 300 Tagen im Jahr gewährleistetist, um dem jahreszeitlich unterschiedlichen Wanderungsverhalten der Fische gerecht zu werden.

\section{Pilotprojekt am Neckar: Fischaufstiegsanlage Lauffen}

Wie eingangs berichtet, ist die ökologische Durchgängigkeit bis zum Jahr 2027 an 


\section{Autorenfassung}

Heinzelmann, Weichert, Wassermann: Hydraul ische Untersuchungen zum Bau einer Fischaufstiegsanlage in Lauffen am Neckar, 2013

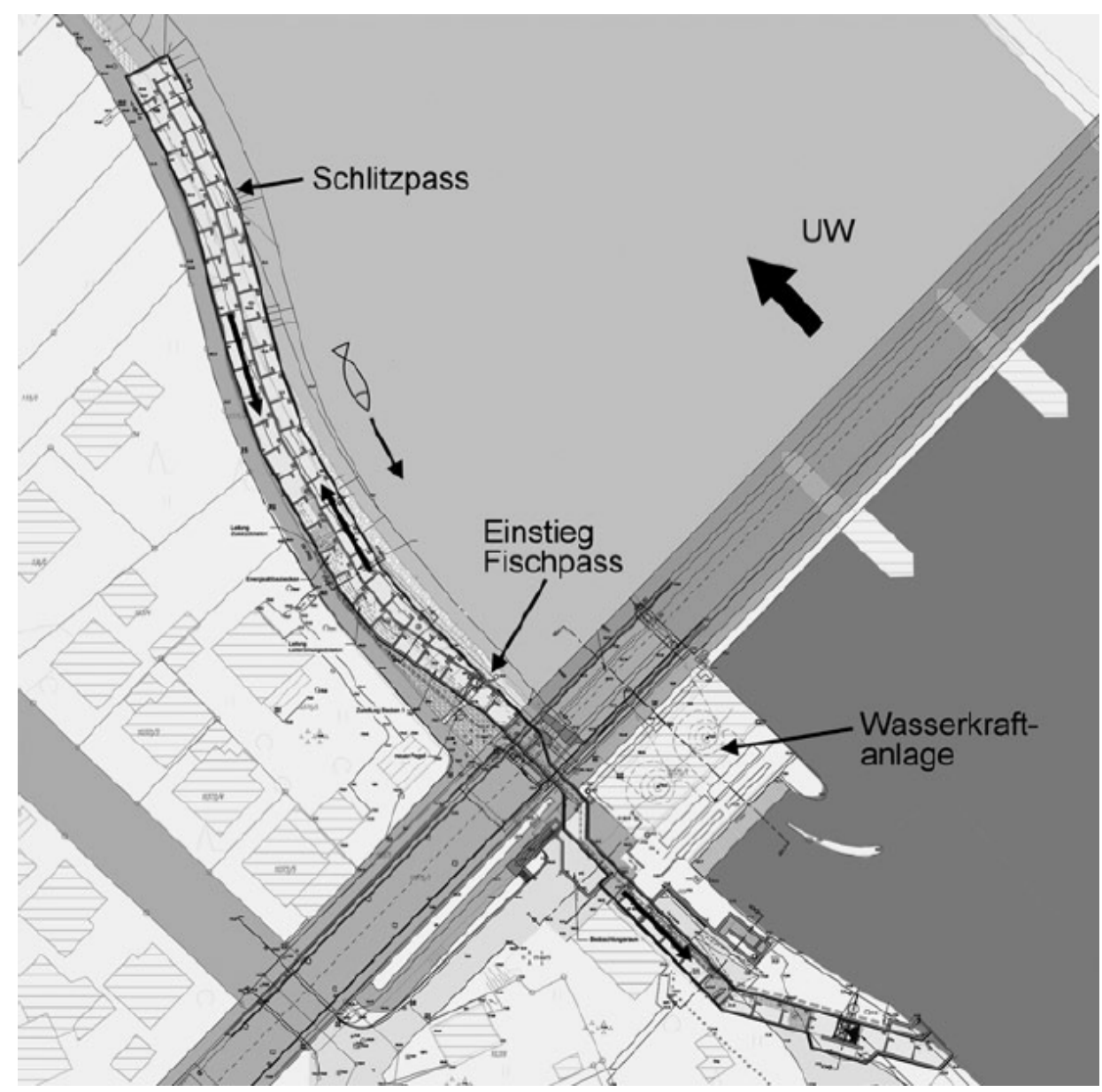

Bild 2: Planungsentwurffür den Schlitzpass Lauffen (Quelle: Ingenieurbüro Floecksmühle, modifiziert)

\section{Heinzelmann, Weichert, Wassermann: Hydraulische Untersuchungen zum Bau einer Fischaufstiegsanlage in Lauffen am Neckar. WasserWirtschaft 1/2 (2013), S. 26-32.}

etwa 250 Stauanlagen der WSV wiederherzustellen. Die Herausforderung für die WSV besteht also darin, trotz der skizzierten Wissenslücken binnen kurzer Zeit eine große Zahl funktionsfähiger FAA zu errichten. Die Handlungsstrategie sieht deshalbvor, dass zunächst Pilotanlagen geplant und gebaut werden, mit deren Hilfe die Wirksamkeit der Anlagen überprüft werden kann. Diese Überprüfung muss neben der rein technischen Funktionskontrolle vor allem auch eine biologische Qualitätssicherung enthalten.

Eine der Pilotanlagen soll an der Neckarstaustufe Lauffen errichtet werden. Die Staustufe umfasst eine Doppelschleusenanlage, eine dreifeldrige Wehranlage sowie ein Wasserkraftwerk. Die Fallhöhe beträgt 8,17 m. Eine FAA existiert derzeit nicht und soll am in Blickrichtung rechten Ufer als Schlitzpass gebaut werden (Bild 1). Die Randbedingungen für den Bau zeichnen sich durch eine 


\section{Autorenfassung}

Heinzelmann, Weichert, Wassermann: Hydraul ische Untersuchungen zum Bau einer Fischaufstiegsanlage in Lauffen am Neckar, 2013

dichte Uferbebauung aus. Darüber hinaus steht die Stauanlage unter Denkmalschutz und die Straßenbrücke soll erneuert werden. Das Kraftwerk ist mit zwei Kaplan-Turbinen (Laufraddurchmesser: 2,78 m) für einen Ausbaudurchfluss von insgesamt $80 \mathrm{~m}^{3} / \mathrm{s}$ ausgelegt. Kennzeichnend für die hydraulischen Verhältnisse unterhalb des Kraftwerks sind besonders ausgeprägte Turbulenzerscheinungen.

Die Dimensionierungeiner FAA richtet sich vorwiegend nach der potenziell natürlichen Fischfauna des Flusssystems, d. h. nach den Fischarten, die im Flusssystem heimisch sind oder die wieder angesiedelt werden sollen. Fürden Stand ort Lauffen sind die empfohlenen Indikatorfischarten: Barbe, Nase, Ukelei, Aal, Brachse, Rotauge, Gründling, Groppe und Meerforelle [5]. Eine Sonderrolle nimmt dabei der Maifisch ein, der auch beim Passieren einer FAA ein Schwarmverhalten zeigt, mit der Folge, dass die geometrischen Abmessungen der Anlage großzügig gewählt werden müssen.

Die in Bild 2 dargestellte FAA wurde im Auftragdes Amtes für Neckarausbau Heidelberg von einem Ingenieurbürogeplant. Die Planung siehteine Beckenlänge von 4,05 m und eine Beckenbreite von 3,20 m vor. Die Schlitzbreite zwischen zwei Becken soll 0,50 m, die Beckentiefe ca. 1,00 m betragen. Wesentlich für die Passierbarkeit des Schlitzpasses sind die Fließgeschwindigkeiten im Bereich der Schlitze zwischen zwei benachbarten Becken. Die maximal zulässige Fließgeschwindigkeit leitet sich aus der Leistungsfähigkeit der Fische ab, die die Anlage passieren sollen. Die Fließgeschwindigkeit im Schlitz stellt sich in Abhängigkeit vom Höhenunterschied zwischen den Becken ein, der im Fall von Lauffen 0,12 m nicht überschreiten darf. Hieraus ergeben sich 67 Becken mit einer Gesamtlänge der FAA von mehr als 270 m. Der erforderliche Durchfluss im Schlitzpass beträgt 660 l/s.

Die Aufgabe von BAW und BfG besteht nun darin, die Planung des Ingenieurbüros unter hydraulischen und biologischen Aspekten zu überprüfen und zu optimieren.

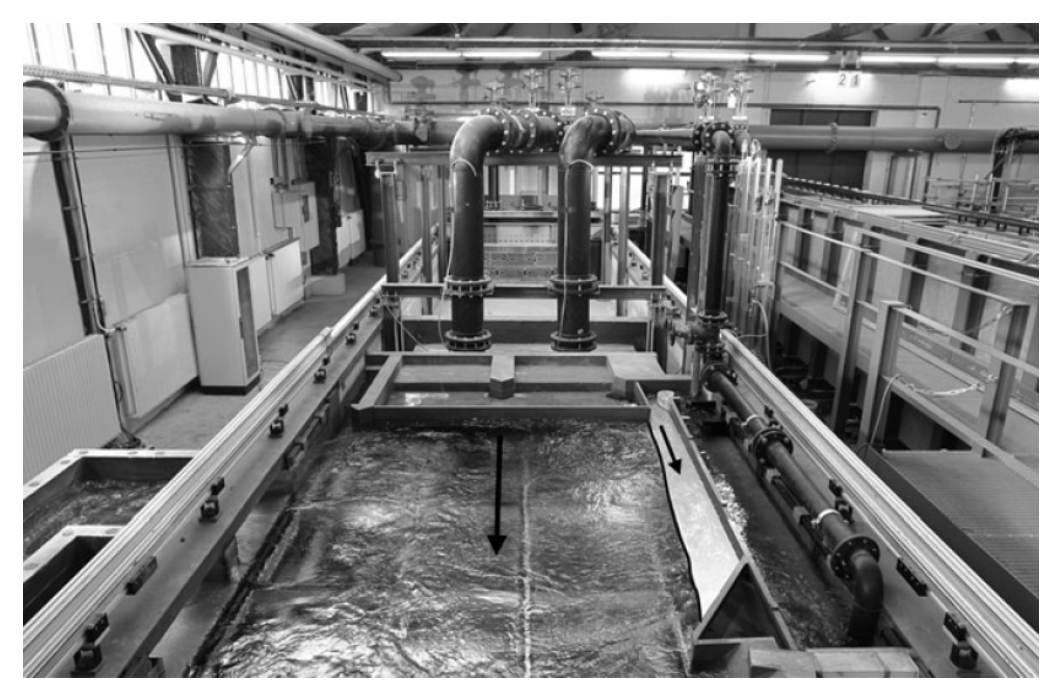

Bild 3: $\quad$ Physikalisches Modell des UnterwassersLauffen: Blick von unterstrom auf den Kraftwerksauslass, rechts der Einstiegin die FAA 


\section{Autorenfassung}

Heinzelmann, Weichert, Wassermann: Hydraul ische Untersuchungen zum Bau einer Fischaufstiegsanlage in Lauffen am Neckar, 2013

\section{Hydraulische Untersuchungen der BAW}

\subsection{Auffindbarkeit}

Der Aspekt der Auffindbarkeit einer FAA beinhaltet im Wesentlichen die Fragestellung, ob wanderwillige Fische, die direkt bis in das Unterwasser einer Staustufe gelangen, in der Lage sind, den Einstieg in die FAA ohne größere Zeitverzögerung zu finden. Hierzu sind vor allem Kenntnisse über das artspezifische Wanderverhalten von Fischen erforderlich. Auch wenn diesbezüglich derzeit noch Wissenslücken existieren, kann den Strömungsverhältnissen im Unterwasser einer Staustufe

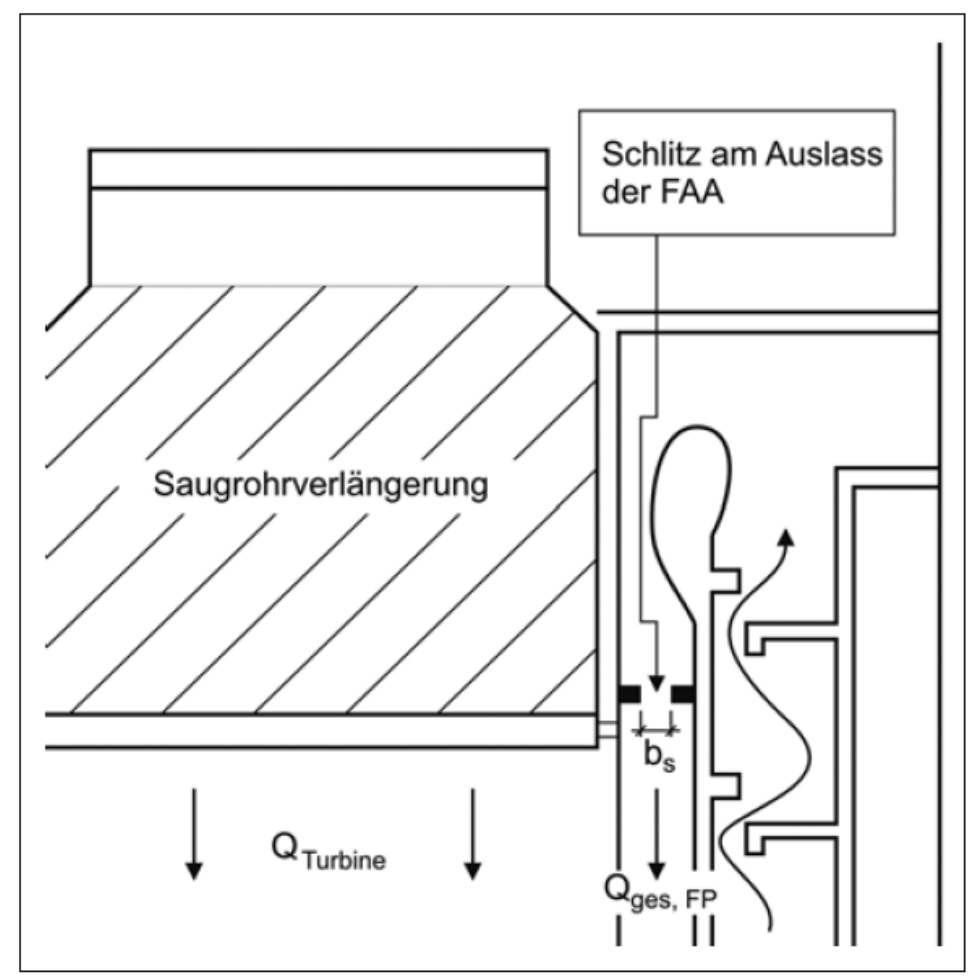

Bild 4: $\quad$ Ausgestaltung des Einstiegs in die FAA 


\section{Autorenfassung}

Heinzelmann, Weichert, Wassermann: Hydraul ische Untersuchungen zum Bau einer Fischaufstiegsanlage in Lauffen am Neckar, 2013

Heinzelmann, Weichert, Wassermann: Hydraulische Untersuchungen zum Bau einer Fischaufstiegsanlage in Lauffen am Neckar. WasserWirtschaft 1/2 (2013), S. 26-32.
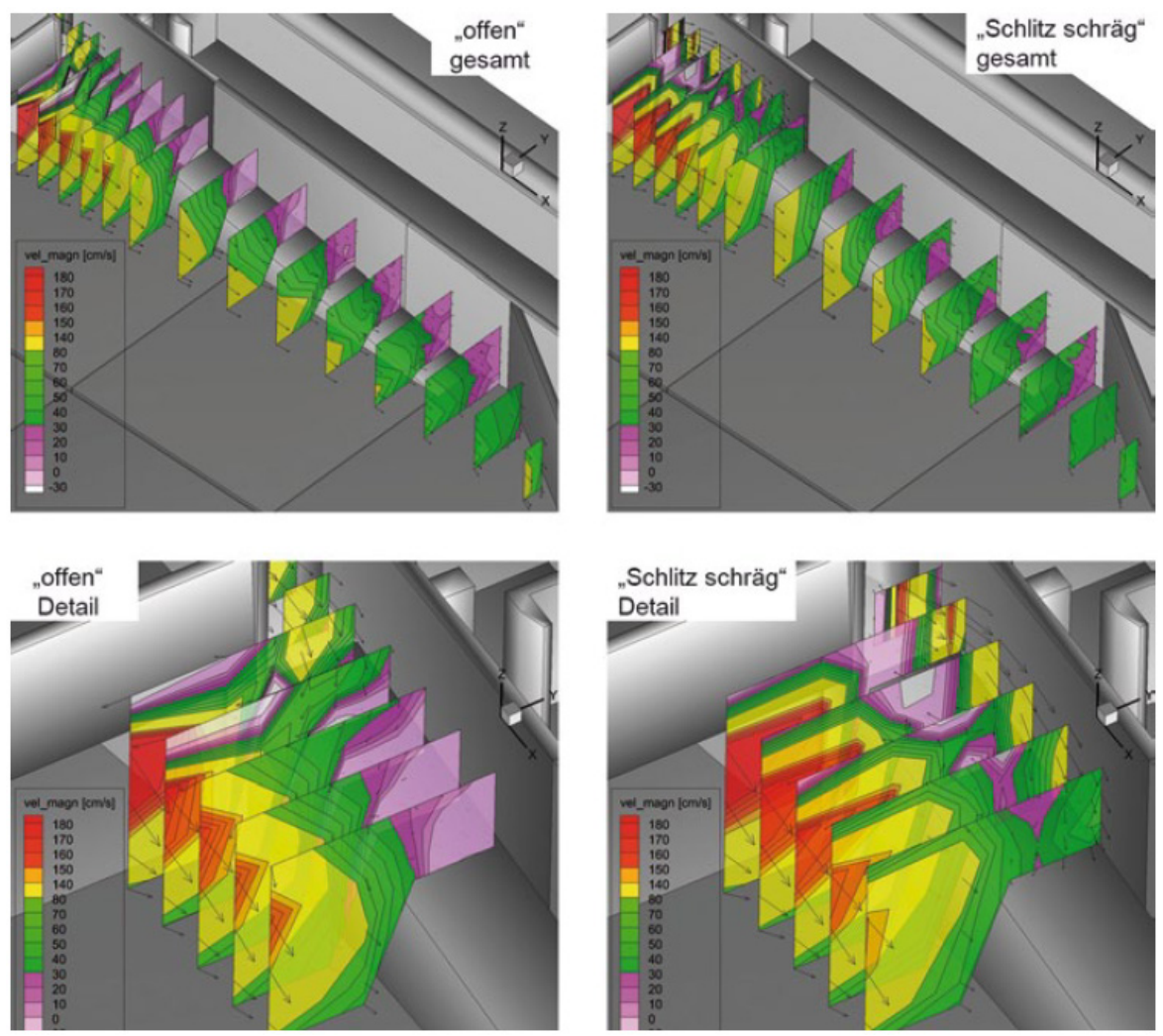

Bild 5: $\quad$ Fließgeschwindigkeiten im physikalischen Modell, Blickvon unterstrom aufden Einstiegsbereich der FAA (Ausgangs- und Vorzugsvariante)

eine zentrale Bedeutung für die Orientierung der Fische zugeschrieben werden. Nur wenn es gelingt, dem Fisch eine für ihn wahrnehmbare Leitströmung aus der FAA zur Verfügung zu stellen, ist die Voraussetzung gegeben, dass die Mehrzahl der wanderwilligen Fische den Einstieg in die FAA findet. Die besondere Herausforderung an Bundeswasserstraßen besteht darin, dass die Strömung im Auslaufbereich des Kraftwerks (Hauptströmung) so dominant ist, dass eine für den Fisch wahrnehmbare Leitströmung aus der FAA nur schwer zu realisieren ist.

Um diese Frage für den Standort Lauffen beantworten zu können, werden in der BAW hydraulische Untersuchungen auf Basis von Naturmessungen sowie numerischen und physikalischen Modellversuchen durchgeführt. In einem ersten Schrittwurden hierfür die Strömungsverhältnisse im Unterwasser der Stauanlage vermessen und der Ist-Zustand in den Modellen abgebildet. Ziel der Modelluntersuchungenist es, die Strömungssituation so zu beeinflussen, dass ein durchgehender und der Leistungsfähigkeit der Fischeangepasster Wanderkorridor geschaffen wird. Dieses Ziel soll 


\section{Autorenfassung}

Heinzelmann, Weichert, Wassermann: Hydraul ische Untersuchungen zum Bau einer Fischaufstiegsanlage in Lauffen am Neckar, 2013

erreicht werden, indem die bauliche Gestaltung des Einstiegsbereichs der FAA sowie das Abflussverhältnis zwischen FAA und Kraftwerk optimiert sowie mit den betrieblichen Randbedingungen der Wasserkraftanlage abgestimmt werden. Nachfolgend werden die physikalischen Modelluntersuchungen näher vorgestellt. Die Naturmessungen sind in [6], [7] beschrieben.

Für die Modelluntersuchungen zur Auffindbarkeit der FAA wurde in der BAW ein physikalisches Modell im Maßstab 1:10 aufgebaut und mit Hilfe der Naturmessungen kalibriert (Bild 3). Besonderes Augenmerk wurde dabeiauf die korrekte Wiedergabe der Strömungssituation im Einstiegsbereich der geplanten FAA gelegt. Hier spielt vor allem die aus den Saugrohren des Kraftwerks austretende, drallbehaftete Strömung eine wichtige Rolle, die im physikalischen Modell durch die Anordnung eines verstellbaren Propellerrades in den Wasserzuleitungen des Modells erreicht wurde. Im Anschluss an die Kalibrierungsphase wurde der Entwurf des Planungsbüros überprüft und optimiert. Wesentliches Element des Entwurfs ist die Verlängerung des Saugrohrs des Kraftwerks (Bild 3 und Bild 4). Diese Maßnahme vermindert einerseits die Turbulenz der aus dem Saugrohr austretenden Strömung und verbessert damit die Leitströmung aus der FAA. Andererseits kann mit der Verlängerung des Saugrohrs der FAA-Einstieg auf der Höhe des Kraftwerksauslasses angeordnet werden, was die Auffindbarkeit deutlich erleichtert. Neben der Analyse der hydraulischen Wirkung der Saugrohrverlängerungsollten durch die Modelluntersuchungen weitere bauliche Komponenten optimiert werden, wie beispielsweise die Ausgestaltung eines Schlitzes am unteren Ende der FAA (Bild 4) sowie die Gestaltung des Übergangs zwischen Gewässersohle und FAA-Einstieg. Zusätzlich stand der für die Ausbildung einer Leitströmung erforderliche Abfluss im Fokus der Untersuchungen. Dieser Abfluss setzt sich zusammen aus dem Abfluss in der FAA und der Zusatzdotation, die im unteren Bereich der FAA hinzugegeben wird. Ziel war es, unter baulich optimierten Randbedingungen den für den Betrieb der Aufstiegsanlage minimal erforderlichen Abfluss zu bestimmen.

Heinzelmann, Weichert, Wassermann: Hydraulische Untersuchungen zum Bau einer Fischaufstiegsanlage in Lauffen am Neckar. WasserWirtschaft 1/2 (2013), S. 26-32.

Die Strömungsgeschwindigkeiten wurden zeitlich und räumlich hoch aufgelöst mit einer ADVSonde (Acoustic Doppler Velocimeter) gemessen, wobei sich die Messungen insbesondere auf die Zone konzentrierten, in der die Kraftwerksströmung und die Strömung aus der FAA zusammentreffen. Für die voraussichtlich ungünstigsten Abflussverhältnisse bei $\mathrm{Q}_{330}$ (Neckar-Abfluss, der an 330 Tagen im Jahr unterschritten wird) wurden neun verschiedene Kombinationen aus Schlitzanordnung und Abfluss aus der FAA analysiert. Die Vorzugsvariante wurde bei zwei weiteren relevanten Abflüssen - an der Untergrenze des Auslegungsbereichs der FAA bei $\mathrm{Q}_{30}$ und bei gerade beginnendem Wehrüberfall bei $\mathrm{Q}_{227}$ - auf Erfüllung der fischökologischen Anforderungen überprüft. Bild 5 zeigt die Ergebnisse für die Ausgangsvariante ohne Einengung der FAA-Öffnung und die Vorzugsvariante mit einem in Strömungsrichtung rechtsseitig angeordneten Schlitz von 0,7 m Breite (Bild 4). 


\section{Autorenfassung}

Heinzelmann, Weichert, Wassermann: Hydraul ische Untersuchungen zum Bau einer Fischaufstiegsanlage in Lauffen am Neckar, 2013

Weitere Randbedingungen zu den beiden in Bild 5 dargestellten Varianten sind Tabelle 1 zu entnehmen.

In Tabelle 1 bezeichnet $\mathrm{Q}_{\text {Turb }}$ den durch das Kraftwerk abgeführten Abfluss. Qges,FAA setzt sich zusammen aus dem Abfluss in der FAA und der Zusatzdotation, d. h. es ist der Abfluss, der am unteren Ende der FAA austritt. Mit $b_{s}$ wird die Breite des Schlitzes am unteren Ende der FAA bezeichnet, vs ist die im Bereich des Schlitzes gemittelte Fließgeschwindigkeit. Die Lage des Schlitzes ist in Fließrichtung angegeben. Der Unterwasserstand ist für beide Varianten identisch.

\begin{tabular}{|l|l|l|l|l|l|l|}
\hline Varianten & QTurb & Qges,FAA & $\mathrm{b}_{\mathrm{s}}$ & $\mathrm{v}_{\mathrm{s}}$ & Lage Schlitz & UW-Stand \\
\hline offen & $80 \mathrm{~m}^{3} / \mathrm{s}$ & $3,0 \mathrm{~m}^{3} / \mathrm{s}$ & $1,65 \mathrm{~m}$ & $0,9 \mathrm{~m} / \mathrm{s}$ & $\begin{array}{l}\text { Keine Ein- } \\
\text { engung }\end{array}$ & $\begin{array}{l}162,64 \mathrm{~m} \text { ü. } \\
\mathrm{NN}\end{array}$ \\
\hline $\begin{array}{l}\text { Schlitz } \\
\text { schräg }\end{array}$ & $80 \mathrm{~m}^{3} / \mathrm{s}$ & $2,1 \mathrm{~m}^{3} / \mathrm{s}$ & $0,7 \mathrm{~m}$ & $1,5 \mathrm{~m} / \mathrm{s}$ & $\begin{array}{l}\text { rechts, } \\
\text { schräg }\end{array}$ & $\begin{array}{l}162,64 \mathrm{~m} \text { ü. } \\
\text { NN }\end{array}$ \\
\hline
\end{tabular}

Tab. 1: $\quad$ Randbedingungen für die Variantenuntersuchungen

Der aus der FAA austreten de Abfluss ergibt sich aus den Schlitzdimensionen und der vorgegebenen Geschwindigkeitim Schlitzvon 1,5 m/s. Sämtliche Größen in Tabelle 1 sind im Naturmaßstab angegeben.

Mit Angaben aus der einschlägigen Literatur über die Schwimmfähigkeit von Fischen lassen sich Geschwindigkeitsbereiche definieren, in denen die Verhältnisse für die Fischwanderung günstig oder ungünstig sind. Die Leistungsfähigkeitder Fische ist dabei abhängigvon der Fischart und vom Entwicklungsstadium der Fische. Hervorzuheben ist, dass nicht nur zu große Geschwindigkeiten im Wanderkorridor der Fische die Auffindbarkeit der FAA beeinträchtigen, sondern dass vor allem auch ein fehlender Strömungsimpuls zur Desorientierung der Fische führen kann. Die geeigneten Strömungsgeschwindigkeiten für die am Neckar empfohlenen Indikatorfischarten betragen 0,3 m/s bis 1,6 m/s [2]. Ziel der Modellversuche war es also, Strömungsbedingungen in einem längs des Ufers durchgehenden Wanderkorrid or zu schaffen, die die Auffindbarkeit des Einstiegs in die FAA bestmöglich unterstützen.

In Bild 5 sind die zeitlichen Mittelwerte der gemessenen Fließgeschwindigkeiten für zwei Varianten dargestellt. Die Abbildung zeigt, dass sich die Strömungsfelder im Einstiegsbereich in Abhängigkeit der geometrischen Ausgestaltungdes Schlitzes und des Abflusses am unteren Ende der FAA signifikant unterscheiden. Für die Variante mit Einstiegsöffnung auf voller Breite (Bild 5, „offen“) ergibt sich, dass die Strömungsgeschwindigkeiten im Nahbereich der FAAnicht ausreichen, um eine Leitwirkung auf die Fische entfalten zu können, da die Leitströmungnach Austritt aus der FAA-Öffnung schon nach wenigen Metern von der Strömung aus dem Kraftwerk dominiert wird. Sohlennah wandernde Fische können auf Grund geringer Geschwindigkeiten über der Sohlenrampe den Einstieg 


\section{Autorenfassung}

Heinzelmann, Weichert, Wassermann: Hydraul ische Untersuchungen zum Bau einer Fischaufstiegsanlage in Lauffen am Neckar, 2013

höchstens zufällig auffinden. Die Verengung des Austrittsquerschnitts, d. h. die Anordnung einer weiteren Trennwand mit Schlitz, erzeugt zwar höhere Fließgeschwindigkeiten im Schlitz, und bei Anordnung in Fließrichtung links bis mittig auch über der Rampe, führt aber gleichzeitig zu großen Strömungsgebieten mit geringen Geschwindigkeiten in der Verlängerung des Kraftwerkspfeilers zwischen FAA und angrenzendem Saugrohr. Die Ergebnisse für die aus neun unter

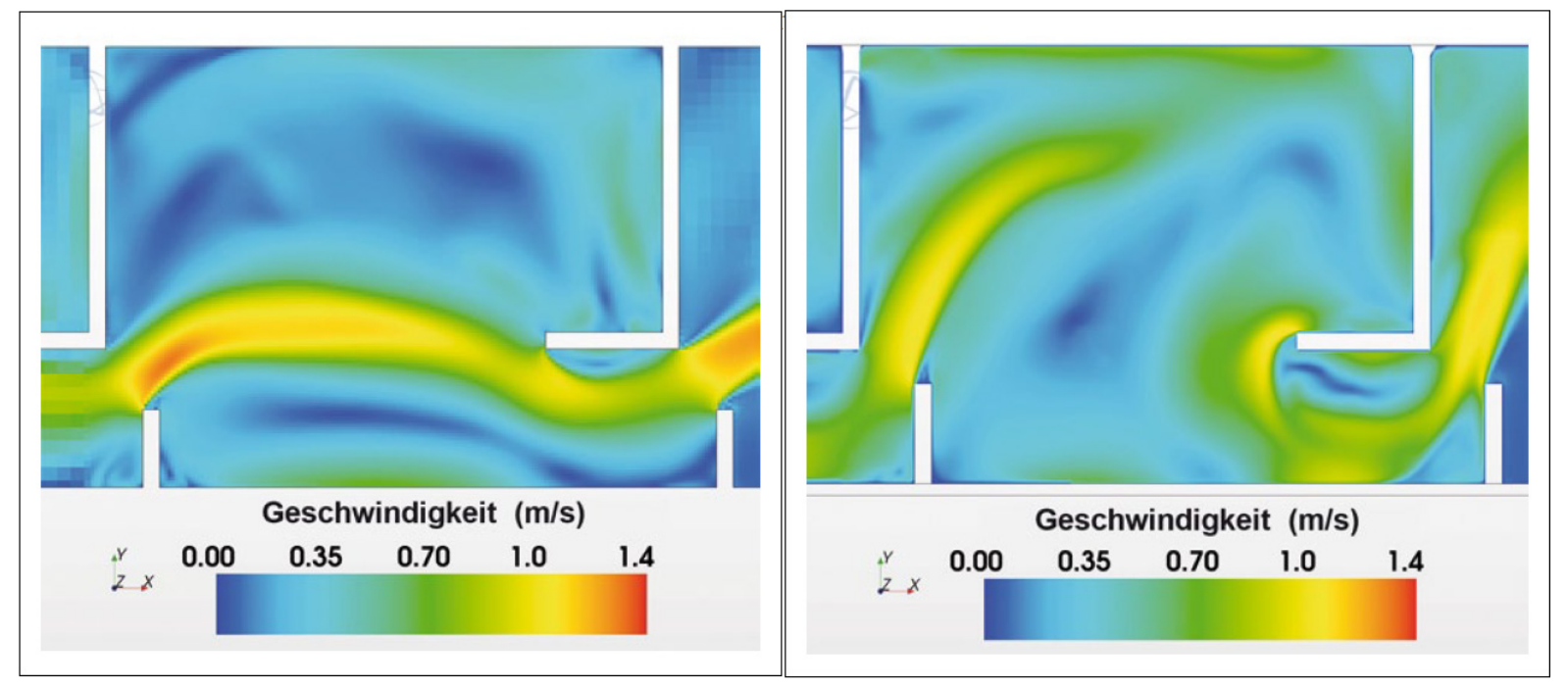

Bild 6: $\quad$ Ergebnisse einer 3-D-HN-Modellierung der Schlitzpassströmung

Heinzelmann, Weichert, Wassermann: Hydraulische Untersuchungen zum Bau einer Fischaufstiegsanlage in Lauffen am Neckar. WasserWirtschaft 1/2 (2013), S. 26-32.

suchten Schlitz- bzw. Abflusskonfigurationen entwickelte Vorzugsvariante zeigt die rechte Hälfte von Bild 5 (Bild 5, „Schlitz schräg“). In der Vorzugsvariante lenkt ein in Fließrichtung rechtsseitig angeordneter Schlitz mit Breite von 0,7 m die Strömung aus der FAA schräg gegen das linke Ufer, wo sie sich zunächst entlang der FAA-Außenwand, weiter stromabim Bereich der Sohlenrampe als durchgehender Korridor ausbreiten kann. Gleichzeitigbewirkt der gegenüber der Ausgangsvariante erhöhte Abfluss aus der FAA, dass der Bereich mit geringen Geschwindigkeiten im Nachlauf des Pfeilers begrenzt bleibt.

Zusätzlich zu den physikalischen Modelluntersuchungen finden derzeit numerische Simulationen für das Unterwasser der Staustufe Lauffen statt. Diese werden mit einem 3-D-Navier-Stokes-Löser durchgeführt, der die freie Wasseroberfläche berechnet und auf Basis von Hexaedergittern arbeitet. Diese Simulationen sind wünschenswert, da mittels der physikalischen Modellversuche zwar die hydraulische Optimierung der baulichen Komponenten erreicht werden kann, die Vermessung des Strömungsfelds im Unterwasser jedoch nur punktuell und mit einem relativ hohen Aufwand mög- 


\section{Autorenfassung}

Heinzelmann, Weichert, Wassermann: Hydraul ische Untersuchungen zum Bau einer Fischaufstiegsanlage in Lauffen am Neckar, 2013

lich ist. Dies hat zur Folge, dass sich die Strömungsparameter aus den Messungen des physikalischen Modells nur statistisch beschreiben lassen.

Auch wenn mit dem numerischen Modell ebenfalls nur eine Annäherung an die realen Verhältnisse möglich ist, ist man im Vergleich zu den Ergebnissen aus dem physikalischen Modell in der Lage, einen weiteren Aspekt zu untersuchen: Liegt eine auf Basis der physikalischen Modelluntersuchungen optimierte Variante vor, so bietet die numerischeSimulation die Möglichkeit, für einen gegebenen Zeitpunkt ein räumlich hoch aufgelöstes Bild der Strömungsvektoren nach Größe und Richtung zu erhalten. Mit dieser Information lässt sich in einem nächsten Schritt bewerten, ob zu jedem Zeitpunkt und für die gegebenen Randbedingungen (z. B. Abflussmengenaufteilung zwischen FAA und Kraftwerk oder Turbinenmanagement) ein durchgehenderWanderkorridor für die Fische zur Verfügung steht. Zusammen mit den Ergebnissen aus den physikalischen Modellversuchen lassen sich damit die hydraulischen Verhältnisse hinsichtlich ihrer Eignung für die Fischwanderung bestmöglich beurteilen.

\subsection{Passierbarkeit}

Neben der Auffindbarkeit spieltfür die Wirksamkeit einer FAA auch die Passierbarkeit der Anlage eine entscheidende Rolle. Wie in Abschnitt 3 bereits erläutert, sieht der Planungsentwurf für den Fischaufstieg an der Stauanlage Lauffen wegen der beengten Platzverhältnisse einen Schlitzpass vor. Dieser weltweit häufig eingesetzte Typ einer beckenartigen FAA wurde bereits verschiedentlich im Rahmen von Forschungsarbeiten, z. B. [8], [9], [10] näher untersucht. Die Untersuchungen zeigten $u$. a., dass sich in Abhängigkeit der geometrischen Anordnung der Schlitze verschiedene Strömungsmuster in den Becken zwischen den Schlitzen einstellen. Wie genau dieses Strömungsfeld beschrieben werden kann (Geschwindigkeiten, Wirbelgrößen) und vor allem, welche Wirkung die verschied enen Strömungsmuster auf die unterschiedlichen wanderwilligen Fischarten haben, ist jedoch noch weitestgehend ungeklärt.

Um das Strömungsfeld näher zu untersuchen, wurde in der BAW ein physikalisches Modell eines Schlitzpasses im Maßstab 1:4 aufgebaut. In diesem Modell sollen in Abhängigkeit verschiedener geometrischer Anordnungen die Strömungsverhältnisse zeitlich und räumlich hoch aufgelöst erfasst werden. Das Modell besteht aus neun aufeinander folgenden Becken, die durch eine Breite von 0,78 m, eine Länge von 0,99 m und eine Sohlenneigung von 2,8 \% gekennzeichnet sind. Die Becken sind so gestaltet, dass die Beckengeometrie flexibel angepasst und optimiert werden kann. Die Strömung innerhalb eines einzelnen Beckens und im Bereich des Schlitzes zwischen zwei Becken soll vermessen und mittels geeigneter hydraulischer Größen als Funktion des Abflusses und der geometrischen Beckendimensionen beschrieben werden. Hierzu soll als optisches Messverfahren ein PIV-System (Particle Image Velocimetry) eingesetzt werden, mit dem die Strömung berührungslos durch den Rinnenboden und die Seitenwände aufgenommen werden kann. Das Messsystem ist dabei so aufgebaut, dass ein synchronisierter Messbetrieb mit dem PIV-System und zwei ADV-Sonden möglich ist. Die Messfrequenz des PIV-Systems 


\section{Autorenfassung}

Heinzelmann, Weichert, Wassermann: Hydraul ische Untersuchungen zum Bau einer Fischaufstiegsanlage in Lauffen am Neckar, 2013

\section{Heinzelmann, Weichert, Wassermann: Hydraulische Untersuchungen} zum Bau einer Fischaufstiegsanlage in Lauffen am Neckar. WasserWirtschaft 1/2 (2013), S. 26-32.

beträgt max. $200 \mathrm{~Hz}$, der Messbereich hat eine Größe von ca. 45 x $40 \mathrm{~cm}$. Einzelheiten hierzu sind in [11] zu finden.

Die Untersuchungen im physikalischen Modell erfüllen neben den gewonnenen Erkenntnissen über das Strömungsfeld einen weiteren Zweck. Die hoch aufgelösten Daten der Strömungsmessungen dienen dazu, die Berechnungsergebnisse der eingesetzten numerischen Modelle zu plausibilisieren und die Modellverfahren weiterzuentwickeln. Wenn sichergestellt ist, dass die numerischen Modellverfahren das Strömungsfeld mit hinreichender Genauigkeit berechnen können, besteht die Möglichkeit, mit geringem Aufwand die Auswirkungen baulicher Varianten zu untersuchen und zu bewerten. Beispielhaft sind in Bild 6 die Resultate einer numerischen 3-D-Modellierung in einem Becken eines Schlitzpasses dargestellt. Die Bilder verdeutlichen den Einfluss der Anordnung der baulichen Elemente auf das Strömungsfeld. Während im linken Bild noch ein durchgehender Strömungsstrahl innerhalb des Beckens zu erkennen ist, zeigt das rechte Bild, dass bereits eine geringfügige Verlängerung und Versatz des Umlenkelements unter Erhaltung der Schlitzbreitehinter dem Schlitz dazu führt, dass der Strömungsstrahl in das Becken abgelenkt wird und so eine durchgehende Strömungssignatur innerhalb des Beckens zu Gunsten einerverbesserten Energiedissipation nicht mehr gegeben ist.

\section{Autoren}

Prof. Dr.-Ing. Christoph Heinzelmann

Dr. sc. techn. Roman Weichert

Dipl.-Met. Stefanie Wassermann

Bundesanstalt für Wasserbau

Kußmaulstr. 17

76187 Karlsruhe

christoph.heinzelmann@baw.de

roman.weichert@baw.de

stefanie.wassermann@baw.de 


\section{Autorenfassung}

Heinzelmann, Weichert, Wassermann: Hydraul ische Untersuchungen zum Bau einer Fischaufstiegsanlage in Lauffen am Neckar, 2013

\section{Christoph Heinzelmann, Roman Weichert and Stefanie Wassermann}

\section{Hydraulic Investigations for the Construction of a Fishway in Lauffen at the River Neckar}

As service provider for the Federal Waterways and Shipping Administration (Wasser- und Schifffahrtsverwaltungdes Bundes, WSV) the Federal Waterways Engineering and Research Institute (Bundesanstalt für Wasserbau, BAW) provides advice for the offices of the WSV regarding the river continuity of Federal waterways. In this context, the investigations of BAW concentrate on hydraulic and waterways engineering aspects. This article reports on current investigations for the construction of a fishway at the barrage Lauffen, River Neckar. Field investigations as well as physical and numerical model simulations are used.

Кристоф Хайнцельманн, Роман Вайхерт и Штефани Вассерманн

\section{Гидравлические исследования для строительства рыбоподъемного сооружения на гидроузле Лауффен (Lauffen) на реке Неккар}

Федеральное управление по гидросооружениям (BAW) является организацией, предоставляющей услуги по техническим и научным вопросам для федерального управления водными путями, водоснабжением, судоходством (WSV); в частности, отделы и ведомства федерального управления водными путями, водоснабжением и судоходством (WSV) могут получить консультации по вопросам проходимости водных путей для рыб. При этом основное внимание в исследованиях Федерального управления по гидросооружениям (BAW) уделяется таким аспектам как гидравлика и гидросооружения. В статье представлены ведущиеся на данный момент исследования для строительства рыбоподъемного сооружения на гидроузле Лауффен (Lauffen) на реке Неккар. Используются не только естественнонаучные исследования, применяются также методы физического и цифрового моделирования.

\section{Literatur}

[1] Bundesanstaltfür Gewässerkunde (Hrsg.): Herstellung der Durchgängigkeit an Staustufen von Bundeswasserstraßen - Fischökologische Einstufungder Dringlichkeit von Maßnahmen für den Fischaufstieg. BfG-Bericht (2010), Nr. 1697.

[2] Deutsche Vereinigung für Wasserwirtschaft, Abwasser und Abfall e.V. (Hrsg.): Fischaufstiegsanlagen und fischpassierbare Bauwerke - Gestaltung, Bemessung, Qualitätssicherung. In: DWA-Merkblätter (2010), Nr. 509 (Entwurf). 


\section{Autorenfassung}

Heinzelmann, Weichert, Wassermann: Hydraul ische Untersuchungen zum Bau einer Fischaufstiegsanlage in Lauffen am Neckar, 2013

[3] Ministerium für Umwelt und Naturschutz, Landwirtschaft und Verbraucherschutz NRW (Hrsg.): Handbuch Querbauwerke. 2005.

[4] Arcadis (Hrsg.): Internationale Leitlinien zum Bau von Fischaufstiegsanlagen. Essen, 2012 (Veröffentlichung auf BAW-Homepage baw.de).

[5] Bundesanstaltfür Gewässerkunde(Hrsg.): Standardisierung der faunistischen und strömungstechnischen Anforderungen an Fischaufstiege am Neckar. Abschlussbericht der Projektgruppe. BfG-Bericht (2011), Nr. 1699.

[6] Heinzelmann, Ch.; Weichert, R.: Wiederherstellung der ökologischen Durchgängigkeit an Bundeswasserstraßen. In: DresdnerWasserbauliche Mitteilungen (2012), Heft 47, S. 205-216.

[7] Sokoray-Varga, B.; Weichert, R.; Lehmann, B.: Flow investigations for fish pass Lauffen/Neckar in field and laboratory. In: Dresdner Wasserbauliche Mitteilungen (2011), Heft 45, S. 87-94.

[8] Wu, S.; Rajaratnam, N.; Katopodis, C.: Structure of flow in a vertical slot fishway. In: Journal of Hydraulic Engineering 125 (1999), No. 4, S. 351-359.

[9] Liu, M.; Rajaratnam, N.; Zhu, D. Z.: Mean flow and turbulence structure in vertical slot fishways. In: Journal of Hydraulic Engineering 132 (2006), S. 765-777.

[10] Tarrade, L.; Texier, A.; David, L.; Larinier, M.: Topologies and measurements of turbulent flow in vertical slot fishways. In: Hydrobiologia (2008), Vol. 609, S. 177-188.

[11] Sokoray-Varga B.; Weichert, R.; Lehmann, B.; Nestmann, F.: Detecting turbulent eddies by means of PIV in a vertical-slot fish pass. In: Proceedings of the 2nd IAHR Europe Congress, 2012. 